\title{
The Equation of the Set of Natural Numbers Just to Sum
}

\author{
Tulus Nadapdap ${ }^{1}$, Tulus ${ }^{2}$, Opim Salim ${ }^{3}$ \\ ${ }^{1}$ Posgraduate Students Faculty of Mathematics and Natural Sciences Universitas Sumatera Utara, Indonesia \\ ${ }^{2,3}$ Postgraduate Lecturer Faculty of Mathematics and Natural Sciences Universitas Sumatera Utara, Indonesia \\ Corresponding Author: Tulus Nadapdap
}

\begin{abstract}
Systems of equations of the form $\mathrm{X}=\mathrm{Y}+$ $Z$ and $X=C$, in which the unknowns are setsof integers," + " denotes pairwise sum of sets $\mathrm{S}+\mathrm{T}=\mathrm{m}+\mathrm{nm} \mathrm{S}, \mathrm{n} \mathrm{T}$, and $\mathrm{C}$ is an ultimately periodic constant. When restricted to sets of natural numbers, such equations can be equally seen as language equations over a one-letter alphabet with concatenation and regular constants, and it is shown that such systems are computationally universal, in the sense that for every recursive set $\mathrm{S} N$ there exists a system with a unique solution containing $T$ with $S=n 16 n+13$ T. For systems over sets of all integers, both positive and negative, there is a similar construction of a system with a unique solution $S=\{n \mid 16 n \in T\}$ representing any hyper-arithmetical set $\mathbf{S} \subseteq \mathrm{N}$.
\end{abstract}

Keywords: Language equations, Natural numbers, Equations of natural number

\section{INTRODUCTION}

In mathematics and computer science, the sign of the star or the operator Kleene is a unary operation, either on a series of set or the set of symbols or characters. The application of the sign of the star to a set $\mathrm{V}$ is written as $\mathrm{V} *$. It is widely used in the simple statements to determine the character of automata specific which means "zero or more".

An equation with the form $\phi(X 1, \ldots, X n)=$ $\psi(\mathrm{X} 1, \ldots, \mathrm{Xn})$, where the unknown $\mathrm{X} 1$ is a formal language. Symbol $\phi, \psi$ used in the operation of the languages theoretically, the incidence of repetitive language constants as well as the Kleene Star and Boolean operations. It will be aware of the form of the system of equations below:

$\mathrm{X} 1=\phi 1(\mathrm{X} 1, \ldots, \mathrm{Xn})$

$\mathrm{Xn}=\phi \mathrm{n}(\mathrm{X} 1, \ldots, \mathrm{Xn})$

with $\phi \mathrm{i}$ using the constants of single and combined operations.

According to Okhotin (2001) combined, the incidence of recurrent and constant single definition for the relationship of grammar. If the relationship is fulfilling, then the equation of the character to be a continuation of the relations of grammar known as the grammar liaison which has the expression of power.

The general form of the expression power of the equation of the language is expressed as follows:

$\phi 1(\mathrm{X} 1, \ldots, \mathrm{Xn})=\psi 1(\mathrm{X} 1, \ldots, \mathrm{Xn})$

$\phi \mathrm{m}(\mathrm{X} 1, \ldots, \mathrm{Xn})=\psi \mathrm{m}(\mathrm{X} 1, \ldots, \mathrm{Xn})$

with $\phi \mathrm{j}, \psi \mathrm{j}$ uses a constant single $\{1\}$ and the merge operation and summation of which has a unique solution with $\mathrm{X} 1=\mathrm{S}$.

These equations will prove that a language may represent a unique solution of a system with repeated occurrences, Boolean operations and constants singular if and only if the language is repetitive. The expression of the power of the same obtained by the use of repeated events with a constant and combined. Later it was found that the equation of the language can be a calculation of the universal without the use of Boolean operations to form the barriers of language $\mathrm{L} \subseteq\{\mathrm{a}, \mathrm{b}\} *$ is the solution of the greatest of the equation $\mathrm{L}=\mathrm{XL}$. 


\section{LITERATURE REVIEW Language Unary}

In computational complexity theory, the language of unary is a formal language in which all the circuit has the form $1 \mathrm{k}$, where " 1 " can be either the symbol remains. For example, the language $\{1,111,1111\}$ is the language of unary. Complexity class of languages is called tally. The equation of the language of the unary with a unique solution does not regularly expressed by Leiss (1994) with equation $X=\phi(X)$ with $\phi$ containing a concatenation, complementation and the constants $\{a\}$.

According to Jez and Okhotin (2009), that the language of the unary can be considered as the set of natural numbers. Equation languages unary in general can be regarded as the equation number with the form of the following equation:

$\mathrm{S}+\mathrm{T}=\{\mathrm{m}+\mathrm{n} \mid \mathrm{m} \in \mathrm{n}, \mathrm{n} \in \mathrm{T}\}$,

is an operation that has been the subject of research in the theory of numbers and kombinatorik.

This paper deals with the equation of the set of natural numbers using only addition and not a Boolean operation, both in the set of natural numbers and the set of integers that are not yet known. The first thing to be considered is the case of the natural numbers to the system of equations is expressed in the following form:

$X \mathrm{Xi} 1+\ldots+\mathrm{Xik}+\mathrm{C}=\mathrm{Xj} 1+\ldots+\mathrm{Xj1}+\mathrm{D}$

variables $(X 1, \ldots, X n)$, where $C, D \in N$ is a constant periodic. In this equation the language $\{a\}$, with the form of the equation: $\mathrm{Xi1} \ldots \mathrm{XikK}=\mathrm{Xj} 1 \ldots \mathrm{Xjl} \mathrm{L}$,

with kostanta $\mathrm{K}, \mathrm{L} \subseteq \mathrm{a} *$. This is the simplest case in equation language. However, as will be demonstrated in this paper, these equations not only have a unique solution is not periodic, but basically can be expressed as a calculation of the universal.

\section{The Equation of A Compilation of A Number Of Native}

At this writing, a compilation of the number of natural $\mathrm{N}=\{0,1,2,3, \ldots\}$ it is assumed pregnancy null. A compilation of the number of $S \subseteq \mathrm{N}$ is periodic if there is a number $\mathrm{d} \geq 0$ and $\mathrm{p} \geq 1$, so $\mathrm{n} \in \mathrm{S}$ if and only if $n+p \in S$ for every $n \geq d$. Note that $S$ is periodic if and only if the appropriate language $\mathrm{L}=\{\mathrm{an} \mid \mathrm{n} \in \mathrm{S}\} \subseteq \mathrm{a} *$ is regular. For each of the two compilation part from the number of $\mathrm{S}, \mathrm{T} \subseteq \mathrm{N}$ is the total from the repertoire of $\{m+n \mid m \in n, n \in T\}$.

\section{Coding Origins}

The $\sigma$ encoding of the natural set of numbers has been found for the set of integers. The first to form is a description of the coding of $\sigma: 2 \mathrm{~N}-\rightarrow 2 \mathrm{~N}$ with a period $\mathrm{p}$ and a single data track $d$, satisfying $p n+d \in$ $\sigma(\mathrm{S})$ if and only if $\mathrm{n} \in \mathrm{S}$, with the track on $\sigma(\mathrm{S})$ independent of S. It is further assumed that each of the tracks must be single, empty, full. This property is expressed in the form of the equation $\mathrm{X}+\mathrm{C}=\mathrm{D}$ which must be checked by coding, the sum $\sigma(\mathrm{S})+$ $\sigma(\mathrm{T})$ must contain the tracks for the joins and for the sums of $\mathrm{S}$ and $\mathrm{T}$, and the sum of the constants $\{0, \mathrm{e}\}$ and $\{0, \mathrm{eJ}\}$ where e, eJ $\geq 1$. This sum must separate the combined tracks and the track counts. Constants $\mathrm{C}$ and $\mathrm{D}$ are also assumed to have a modulo $\mathrm{p}$ structure track.

\section{MATERIAL AND METHODS}

This research is a literature study to identify an equation in the set of natural numbers only against the sum. As for the steps in the completion of this research are: 1). Collect information about the equation on the set of the natural numbers just to sum; 2). Studying the theories related to the original number; 3 ). Discuss the similarities in the set of natural numbers just to sum; 4). Analyze the results of the research that has been studied; 5). Summing up the results of the study of equations in the set of natural numbers only against the sum.

\section{Encoding The Set Of}

The concept of operation is the sum set of a certain shape that has the structure of the periodic still provide predictable results when summing the association of one with the other set. Written in a structure is a set of singular of original number and 
the number of encoding the set of effectively produce combined and the number of encoding the set. Suppose $\sigma: 2 \mathrm{~N}$ $\rightarrow 2 \mathrm{~N}$ be a function mapping the set $\mathrm{S} \subseteq \mathrm{N}$ for encoding $\sigma(\mathrm{S})$. A set of numbers $\mathrm{T} \subseteq \mathrm{N}$ will be represented by another set $\mathrm{S} \subseteq \mathrm{N}$, containing numbers $16 n+13$ if and only if $\mathrm{n}$ is a $\mathrm{T}$. The number of members the number $\mathrm{i}$ with i 13 in $\mathrm{S}$ does not depend on $\mathrm{T}$.

Definition 1 (Calkin and Erdos (1965)), i.e. the set $S \subseteq N$ for every $i \in\{0,1, \ldots, 15\}$, $\operatorname{TRACKi}(S)=\{16 n+i \in S\}$ is the set of on the track to the-i from $S$. For every $T \subseteq N$, such as $\operatorname{ti}(T)=\{16 n+i \in T\}$ declare the set of $\mathrm{T}$ on the track-to - $\mathrm{i}$ and others - his is the track empty. A set $S$ is said to have a track empty $\mathrm{i}$ if $\operatorname{TRACKi}(\mathrm{S})=\varnothing$, and a TRACK full if TRACKi(S) $=\mathrm{N}$.

In this case, can be said to be a set of $\mathrm{T}$ must be encoded in track 13 of a set $\mathrm{S}$. The rest of the track $S$ contains the information to complete the form: track 0 , which contains $\{0\}$, tracks $6,8,9$ and 12 full and the rest of the track is empty.

Definition 2 Ordos (1965)), any set $\mathrm{T} \subseteq \mathrm{N}$ is the encoding of the set

$\mathrm{S}=\sigma(\mathrm{T})=\{0\} \cup \tau 6(\mathrm{~N}) \cup \tau 8(\mathrm{~N}) \cup \tau 9(\mathrm{~N}) \mathrm{U}$ $\tau 12(\mathrm{~N}) \cup \tau 13(\mathrm{~N})$.

The nature of the first encoding is stated that there are some similarities, with all encoding the set of force as a solution to the set.

Lemma 1 (Luczak (1995)), a set $\mathrm{S} \subseteq \mathrm{N}$ satisfies the equation:

$$
S+\{0,4,11\}=\bigcup_{i \in\{0,4,8,8,9,10,12,13\}} \tau_{i}(N) \cup \bigcup_{i \in\{1,3,7\}} \tau_{i}(N+1) \cup\{11\}
$$

if and only if $\mathrm{S}=\sigma(\mathrm{T})$ for some $\mathrm{T} \subseteq \mathrm{N}$.

Proof. Suppose $\mathrm{S}$ every collection which satisfies the equation then the sum $S+\{0,4$, $11\}$ has a track empty 2, 5, 14 and 15 .

TRACK2 $(\mathrm{S}+\{0,4,11\})=$ TRACK5 $(\mathrm{S}+$ $\{0,4,11\})=\operatorname{TRACK} 14(\mathrm{~S}+\{0,4,11\})=$ TRACK15 $(S+\{0,4,11\})=\emptyset$.

For this condition, $\mathrm{S}$ must have a lot of track is empty. Exactly, every track $\mathrm{t}, \mathrm{t}+$ $4, t+11$ are in the $\{2,5,14,15\}$ should be a track is empty in S. Total track compilation of the,$\{2,5,14,15\}-\{0,4,11\}=\{1,2,3$,
$4,5,7,10,11,14,15\}$ is the volume track which should be empty in $\mathrm{S}$.

The same considerations occurred to track 11, as TRACK11 $(\mathrm{S}+\{0,4,11\})$ $=\{0\}$. For each track $\mathrm{t}$ with $\mathrm{t}=11, \mathrm{t}+4=11$ or $\mathrm{t}+11=11$ track $\mathrm{t}, \mathrm{S}$ should be the form of track is empty or containing the constants of single-TRACKt $(\mathrm{S})=\{0\}$, the last should have one of t. Now will count all the track $t$ is a track with a number of $\{11\}-\{0,4,11\}$ $=\{0,7,11\}$. Because the track number 7 and 11 are already known to empty, means TRACK0 $(\mathrm{S})=\{0\}$.

To prove that $\mathrm{S}$ is the encoding is valid from some of the repertoire, the rest can be shown that the number $6,8,9,12 \mathrm{~S}$ full. Consideration of the first to TRACK3 $(\mathrm{S}+\{0,4,11\})=\{\mathrm{N}+1\}$. We suppose reckoning track number $\mathrm{t}$, there is a $\mathrm{tJ} \in\{0$, $4,11\}$ with $(\mathrm{t}+\mathrm{tJ})=3$ : $\mathrm{a}\{3\}-\{0,4,11\}=$ $\{3,8,15\}$.

Then track 3, 15 are known to empty, $\mathrm{N}+1=$ TRACK $3(\mathrm{~S}+\{0,4,11\})=$ TRACK3 (S) $\cup$ TRACK15 ((S)+1) U TRACK8 $((S)+1)=\varnothing \cup \emptyset \cup$ TRACK8 $((\mathrm{S})+1)=$ TRACK8 $(\mathrm{S})+1$. with so track 8 from $\mathrm{S}$ full. Very same argument used to prove that track 12, 9, 6 full. With regard TRACK7 $(S+\{0,4,11\})=\{\mathrm{N}+1\} .\{7\}-$ $\{0,4,11\}=\{7,3,12\}$. Then aware that the track 3 and 7 vacant, track 12 full:

$\mathrm{N}+1=$ TRACK7 $(\mathrm{S}+\{0,4,11\})=$ TRACK7 (S) $\cup$ TRACK3 $((\mathrm{S})+1) \quad \cup$ TRACK12 $((\mathrm{S})+1)=\emptyset \cup \emptyset \cup$ TRACK12 $((\mathrm{S})+1)=$ TRACK12 $(\mathrm{S})+1$.

In the same way TRACK9 $(\mathrm{S}+\{0,4,11\})$ $=\mathrm{N}$, then $\{9\}-\{0,4,11\}=\{9,5,14\}$ and track 5, 14 empty, until track 9 full :

$\mathrm{N}=$ TRACK9 $(\mathrm{S})+\{0,4,11\})=$ TRACK9 (S) $\cup$ TRACK5 $(\mathrm{S}) \cup$ TRACK14 $((\mathrm{S})+1)=$ TRACK9 (S) $\cup \emptyset \cup \emptyset=$ TRACK9 (S)

Will now be examined TRACK10 $(\mathrm{S}+\{0$, $4,11\})=N$, then $\{10\}-\{0,4,11\}=\{10,6$, $15\}$ because track 10, 15 blank, track 6 full: $\mathrm{N}=\operatorname{TRACK} 10(\mathrm{~S})+\left\{\begin{array}{l}0,4,11\})= \\ \text { T }\end{array}\right.$ TRACK10 (S) $\cup$ TRACK6 (S) $U$ TRACK15 $((\mathrm{S})+1)=\varnothing \cup$ TRACK6 $(\mathrm{S}) \cup \emptyset$ $=$ TRACK6 $(\mathrm{S})$, thus it is proved that $\mathrm{S}=$ $\sigma(\mathrm{T})$ for $\mathrm{T}=\mathrm{TRACK} 13(\mathrm{~S})$. 


\section{Operation Simulation}

The purpose of this section is to prove the second property of the encoding of $\sigma$, namely that the number of encoding of the two sets and a set of constants is effective on a combination of two of the set. These properties are formally stated in the lemma below, along with the association constant of the actual:

Lemma 2 (Kunc (2007)), for all of the set $\mathrm{X}, \mathrm{Y}, \mathrm{Z} \subseteq \mathrm{N}$

$\sigma(\mathrm{Y})+\sigma(\mathrm{Z})+\{0,1\}=\sigma(\mathrm{X})+\sigma(\{0\})+\{0$, $1\}$ if and only if $Y+Z=X$

and

$\sigma(\mathrm{Y})+\sigma(\mathrm{Z})+\{0,2\}=\sigma(\mathrm{X})+\sigma(\{0\})+\{0$, $2\}$ if and only if $Y \cup Z=X$

Proof. The goal is to show that for all $\mathrm{Y}, \mathrm{Z}$ $\subseteq \mathrm{N} \sigma(\mathrm{Y})+\sigma(\mathrm{Z})+\{0,1\}$

Encoding the set of $\mathrm{Y}+\mathrm{Z}+1$ is a track, while an index of all the other tracks do not depend on Y or Z. Similarly, the number of:

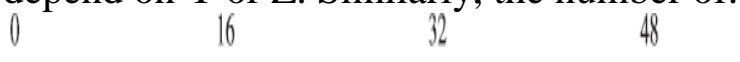

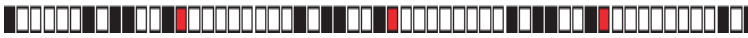
$\sigma(\mathrm{Y})+\sigma(\mathrm{Z})+\{0,2\}$

have a track that encode $\mathrm{Y} \cup \mathrm{Z}$, while the rest does not depend on $\mathrm{Y}$ and $\mathrm{Z}$.

The main part of the sum is $\sigma(\mathrm{Y})+$ $\sigma(Z)$, so that the first will be counted is the equation in the following form:
$\sigma(\mathrm{Y})=\{0\} \cup \tau 6(\mathrm{~N}) \cup \tau 8(\mathrm{~N}) \cup \tau 9(\mathrm{~N}) \cup$ $\tau 12(\mathrm{~N}) \cup \tau 13(\mathrm{~N})(\mathrm{Y})$

and

$\sigma(\mathrm{Y})=\{0\} \cup \tau 6(\mathrm{~N}) \cup \tau 8(\mathrm{~N}) \cup \tau 9(\mathrm{~N}) \cup$ $\tau 12(\mathrm{~N}) \cup \tau 13(\mathrm{~N})(\mathrm{Z})$

the amount of $\sigma(Y)+\sigma(\mathrm{Z})$ is a combination of the 36 track is empty, each in the form of a sum of two track single. Any amount is contained in a track, the single also. For example, the number of track 8 from $\sigma(\mathrm{Y})$ and track 9 of $\sigma(Z)$ are on track $1=8+9$ and the same with

$\tau 8(\mathrm{~N})+\tau 9(\mathrm{~N})=\{8+9+16(\mathrm{~m}+\mathrm{n}) \mid \mathrm{m}, \mathrm{n} \geq$ $0\}=\{1+16 \mathrm{n} \mid \mathrm{n} \geq 1\}=\tau 1(\mathrm{~N}+1)$

while with the added track 13 from $\sigma(\mathrm{Y})$ in track 13 of $\sigma(\mathrm{Y})$ will produce:

$\tau 13(\mathrm{Y})+\tau 13(\mathrm{Z})=\{26+16 \mathrm{~m} \mid \mathrm{m} \in \mathrm{Y}, \mathrm{n} \in$ $\mathrm{Z}\}=\tau 10(\mathrm{Y}+\mathrm{Z}+1)$

shown in table 3.1. Any question mark indicating the track with a value that is not specified. Although these values can be calculated, really not relevant because it does not affect the value of the number of $\sigma(Y)+\sigma(Z)+\{0,1\}$ and $\sigma(Y)+\sigma(Z)+\{0$, $2\}$. What is important is that none of this track contains the constant 0 .

The value of each track i of $\sigma(\mathrm{Y})+$ $\sigma(Z)$ is obtained as the sum of all combined in table 3.1 included on the track-i. The value of the end of the track is presented in the column are presented in table 3.2.

Table 3.1. The number of tracks in $\sigma(Y)+\sigma(Z)$.

\begin{tabular}{ccccccc}
\hline & $0:\{0\}$ & $6: \mathrm{N}$ & $8: \mathrm{N}$ & $9: \mathrm{N}$ & $12: \mathrm{N}$ & $13: \mathrm{Z}$ \\
\hline $0:\{0\}$ & $0:\{0\}$ & $6: \mathrm{N}$ & $8: \mathrm{N}$ & $9: \mathrm{N}$ & $12: \mathrm{N}$ & $13: \mathrm{Z}$ \\
$6: \mathrm{N}$ & $6: \mathrm{N}$ & $12: \mathrm{N}$ & $14: \mathrm{N}$ & $15: \mathrm{N}$ & $2: \mathrm{N}+1$ & $3: ?$ \\
$8: \mathrm{N}$ & $8: \mathrm{N}$ & $14: \mathrm{N}$ & $0: \mathrm{N}+1$ & $1: \mathrm{N}+1$ & $4: \mathrm{N}+1$ & $5: ?$ \\
$9: \mathrm{N}$ & $9: \mathrm{N}$ & $15: \mathrm{N}$ & $1: \mathrm{N}+1$ & $2: \mathrm{N}+1$ & $5: \mathrm{N}+1$ & $6: ?$ \\
$12: \mathrm{N}$ & $12: \mathrm{N}$ & $2: \mathrm{N}+1$ & $4: \mathrm{N}+1$ & $5: \mathrm{N}+1$ & $8: \mathrm{N}+1$ & $9: ?$ \\
$13: \mathrm{Y}$ & $13: \mathrm{Y}$ & $3: ?$ & $5: ?$ & $6: ?$ & $9: ?$ & $10: \mathrm{Y}+\mathrm{Z}+1$ \\
\hline
\end{tabular}

Table 3.2. The number of tracks in $\sigma(Y)+\sigma(Z)$ by a constant

\begin{tabular}{ccccccc}
\multicolumn{5}{c}{ Table 3.2. The number of tracks in $\mathbf{\sigma}(\mathbf{Y})+\boldsymbol{\sigma}(\mathbf{Z})$ by a constant. } \\
\hline & $\sigma(Y)$ & $\sigma(Z)$ & $\sigma(Y)+\sigma(Z)$ & $\sigma(Y)+\sigma(Z)+\{0,1\}$ & $\sigma(Y)+\sigma(Z)+\{0,2\}$ \\
\hline 0 & $\{0\}$ & $\{0\}$ & $\mathrm{N}$ & $\mathrm{N}$ & $\mathrm{N}$ \\
1 & & & $\mathrm{~N}+1$ & $\mathrm{~N}+1$ & $\mathrm{~N}+1$ \\
2 & & & $\mathrm{~N}+1$ & $\mathrm{~N}+1$ & $\mathrm{~N}$ \\
3 & & & $\mathrm{~N}+1$ & $\mathrm{~N}+1$ & $\mathrm{~N}+1$ \\
4 & & & $\mathrm{~N}+1$ & $\mathrm{~N}+1$ & $\mathrm{~N}+1$ \\
5 & $\mathrm{~N}$ & $\mathrm{~N}$ & $\mathrm{~N}$ & $\mathrm{~N}$ & $\mathrm{~N}+1$ \\
6 & $\mathrm{~N}$ & $\mathrm{~N}$ & $\mathrm{~N}$ \\
7 & $\mathrm{~N}$ & $\mathrm{~N}$ & $\mathrm{~N}$ & $\mathrm{~N}$ & $\mathrm{~N}$ \\
9 & $\mathrm{~N}$ & $\mathrm{~N}$ & $\mathrm{~N}$ & $\mathrm{~N}+\mathrm{Z}+1$ & $\mathrm{~N}$ \\
10 & & & $\mathrm{Y}+\mathrm{Z}+1$ & $\mathrm{~N}$ & $\mathrm{~N}$ \\
11 & $\mathrm{~N}$ & $\mathrm{~N}$ & $\mathrm{~N}$ & $\mathrm{~N}$ & $\mathrm{~N} Z$ \\
12 & $\mathrm{Y}$ & $\mathrm{Z}$ & $Y \mathrm{~N}$ & $\mathrm{~N}$ & $\mathrm{~N}$ \\
14 & & & $\mathrm{~N}$ & & \\
15 & & & & &
\end{tabular}

Now the charge contained in the track $\sigma(\mathrm{Y})+\sigma(\mathrm{Z})+\{0,1\}$ can fully

described. The calculation is given in table 3.2 and the result is that for all $\mathrm{Y}$ and $\mathrm{Z}$, 
TRACK11 $(\sigma(\mathrm{Y})+\sigma(\mathrm{Z})+\{0,1\})=\mathrm{Y}+\mathrm{Z}$ $+1$

TRACKi $(\sigma(\mathrm{Y})+\sigma(\mathrm{Z})+\{0,1\})=\mathrm{N}+1$, for $\mathrm{i} \in\{2,3,4,5\}$

TRACKi $(\sigma(Y)+\sigma(Z)+\{0,1\})=N$, for each i

and the equation

$\mathrm{X}=\mathrm{Y}+\mathrm{Z}$ if and only if $\sigma(\mathrm{X})+\sigma(\{0\})+\{0$, $1\}=\sigma(Y)+\sigma(Z)+\{0,1\}$ as $X=X+\{0\}$

For the set of $\sigma(Y)+\sigma(Z)+\{0,2\}$, in the same way for all $\mathrm{Y}$ and $\mathrm{Z}$,

TRACK13 $(\sigma(\mathrm{Y})+\sigma(\mathrm{Z})+\{0,2\})=\mathrm{Y} \cup \mathrm{Z}$ TRACKj $(\sigma(\mathrm{Y})+\sigma(\mathrm{Z})+\{0,1\})=\mathrm{N}+1$, for $\mathrm{j} \in\{1,3,4,5,7\}$

TRACKj $(\sigma(Y)+\sigma(Z)+\{0,1\})=N$, for each $\mathrm{j}$

and therefore, for all $\mathrm{X}, \mathrm{Y}, \mathrm{Z}$ applicable:

$\mathrm{X}=\mathrm{Y} \cup \mathrm{Z}$

if and only if

$\sigma(\mathrm{X})+\sigma(\mathrm{X})+\{0,2\}=\sigma(\mathrm{Y})+\sigma(\mathrm{Z})+\{0$, $1\}$,

because $\mathrm{X}=\mathrm{X} \cup \mathrm{X}$.

\section{The Simulation System Is The Set Of Natural Numbers}

In the operation of the simulation of the individual stated in lemma 2 is valid only on the equations in a simple form, then the task of the first is to convert the given system into a form like the following:

Lemma 3 Oktohin (2001), for each equation of the set of numbers in the variables $(\mathrm{X} 1, \ldots, \mathrm{Xn})$ that uses combined, the sum and constants from a class $\mathrm{C}$ there is a system of equations in variabael $(\mathrm{X} 1, \ldots$, $\mathrm{Xn}, \mathrm{Xn}+1, \ldots, \mathrm{Xn}+\mathrm{m})$ all the equations of the form $\mathrm{Xi}=\mathrm{Xj}+\mathrm{Xk}, \mathrm{Xi}=\mathrm{Xj} \cup \mathrm{Xk}$ or $\mathrm{Xi}=\mathrm{C}$ with $\mathrm{C} \in \mathrm{C}$, se - up of the solution set of the system is $\{(\mathrm{S} 1, \ldots, \mathrm{Sn}, \mathrm{f} 1(\mathrm{~S} 1, \ldots, \mathrm{Sn}), \ldots$, $\mathrm{fm}(\mathrm{S} 1, \ldots, \mathrm{Sn})) \mid(\mathrm{S} 1, \ldots, \mathrm{Sn})$ is a solution of the system which is the original $\}$. For some functions monotonous fi, ..., fm.

Lemma 4 (Sandor (2004), for each equation of the set of natural numbers in the variables $(\mathrm{X} 1, \ldots, \mathrm{Xn})$, with all equations of the form $\mathrm{X}=\mathrm{Y}+\mathrm{Z}, \mathrm{X}=\mathrm{Y} \cup \mathrm{Z}$ or $\mathrm{X}=\mathrm{C}$, there is a system variable $(\mathrm{XJ}, \ldots, \mathrm{XJ}$ ) using only addition and constants $\{0,1\},\{0$, $2\},\{0,4,11\}, \sigma(\{0\})$ and $\sigma(C)$ with a $C$ used in the initial system constants periodic in lemma 1 , so that $\mathrm{XJ}=\mathrm{SJ}$ is the solution of the last system if and only if the system has a solution $\mathrm{X} 1=\mathrm{S} 1$ with $\mathrm{SJ}=\sigma(\mathrm{Si})$.

Proof. The proof is by changing the system according to lemma 1 and 2. First, the new system contains equations that each of the variables $\mathrm{XJ}$ :

$$
X^{\prime}+\{0,4,11\}=\bigcup_{i \in\{0,4,6,8,10,10,12,13\}} \tau_{i}(N) \cup \bigcup_{i \in\{1,3,7\}} \tau_{i}(N+1) \cup\{11\}
$$

Furthermore, for each equation $\mathrm{X}=$ $\mathrm{Y}+\mathrm{Z}$ in the original system there is an equation with the form:

$$
X^{\prime}+\sigma(\{0\})+\{0,1\}=Y^{\prime}+Z^{\prime}+\{0,1\}
$$

in the new system. Similarly, for any equation of the form $\mathrm{X}=\mathrm{Y} \cup \mathrm{Z}$ new system contains equations:

$\mathrm{XJ}+\mathrm{XJ}+\{0,2\}=\mathrm{Y} \mathrm{J}+\mathrm{ZJ}+\{0,2\}$

Finally, any equation $\mathrm{X}=\mathrm{C}$ in the original system is represented in the new system with the following equation:

$\mathrm{XJ}=\sigma(\mathrm{C})$.

By using lemma 1, it can be ensured that every solution of (SJ ,...,SJ ) of the system that was formed to meet $\mathrm{SJ}=\sigma(\mathrm{Si})$ for some $(\mathrm{Si}) \subseteq \mathrm{N}$.

Theorem 3.1 .For each set of repeated $\mathrm{T} \subseteq$ $\mathrm{N}$, there is a system of equations:

$\phi 1(\mathrm{X} 1, \ldots, \mathrm{Xn})=\psi 1(\mathrm{X} 1, \ldots, \mathrm{Xn})$

$\phi \mathrm{m}(\mathrm{X} 1, \ldots, \mathrm{Xn})=\psi \mathrm{m}(\mathrm{X} 1, \ldots, \mathrm{Xn})$

with $\phi \mathrm{i}, \phi \mathrm{j}$ using the operations of addition and constants periodic, which has a unique solution with $X 1=S$ where $S=\{n \mid 16 n+13$ $\in \mathrm{T}\}$.

Precisely, the form of the equation requires constant limited $\{0,1\},\{0,2\},\{0$, $4,11\}$ and a constant infinite $\sigma(\{0\})$ on the encoding of the set used in theorem 3.1 constant infinite $\sigma(\{0\})$ by lemma 2 and the one constant infinite other of lemma 1.

Note that $\mathrm{T}$ is a computation that can be lowered into the $\mathrm{S}$ through the " $16 \mathrm{~N}+$ 13 ", pay attention to the following explanation:

Result 1. For each set of repeated $\mathrm{T} \subseteq \mathrm{N}$, there is a system of equations in the set of numbers by using addition and constants 
periodic has a unique solution, that where the computation is said as a powerful $\mathrm{T}$.

Result 2. There is a system of equations a set of numbers that use penjumla - han and constants periodic has a solution the most.Finally, the solution of the problem system of equations is to be strong in the case of combined and sum.

Theorem 3.2 Testing problems systems of equations the set of original numbers using addition and constants periodic has a solution, namely П0.

These results apply also to the equations of the language over the alphabet with recurrent events as operation with a constant choice.

Result 3. For each iteration language $\mathrm{L} \subseteq$ $\mathrm{a} *$, there is a system of equations languages $\phi 1(\mathrm{X} 1, \ldots, \mathrm{Xn})=\psi 1(\mathrm{X} 1, \ldots, \mathrm{Xn})$ $\phi \mathrm{m}(\mathrm{X} 1, \ldots, \mathrm{Xn})=\psi \mathrm{m}(\mathrm{X} 1, \ldots, \mathrm{Xn})$

with $\phi \mathrm{i}, \phi \mathrm{j}$ using the operation repeated events and constants commonly more than one alphabet, which has a unique solution with $\mathrm{X} 1=\mathrm{LJ}$, where $\mathrm{L}=\{\mathrm{an} \mid \mathrm{a} 16 \mathrm{n}+13 \in \mathrm{LJ}$ \}.

\section{RESULTS AND DISCUSSION The Equation In The Set Of Integers}

The purpose of this section is to get the same result for the equation in the set of integers, namely that the equation with combined and the sum can be simulated by the equation sum just by using some simple coding.

According to the Szemeredi (1975), the equation of the set of integers with the combined and the sum that represents the unique solution of the equation exactly with the set of hyper-aritmatikal. Suppose $\Sigma 1$ group shows the set defined by the existential arithmetic, namely the set:

$\{\mathrm{n} \in \mathrm{Z} \mid \exists X \subseteq \mathrm{Z}: \phi(X, \mathrm{n})\}$

where $\phi$ is a formula of the first group containing a statement $\mathrm{x} \in \mathrm{X}$. Set in $\Pi 1$ defined together with a count of second class.

Meeting $\Delta^{1}=\Sigma_{1}^{1}-\Pi_{1}^{1}$ For each set of hyper-aritmatikal

$\mathrm{S} \subseteq \mathrm{Z}$, there is a system of equations in the set of integers using merge, sum, and constants $\{1\}, \mathrm{N}$ and $-\mathrm{N}$, which has a unique solution $(\mathrm{S}, \ldots)$ and vice versa, if the set of a unique solution of the system of equations in the set of integers that uses the operation in the first group arithmetic, peano, then the set is a hyper-aritmatikal.

The resulting system will be coded into the system only uses addition. The set of integers to be transformed into the case of the natural numbers. For any set $\mathrm{T} \subseteq \mathrm{Z}$ is encoded as a set:

$\mathrm{S}=\sigma(\mathrm{T})=(\{0\}) \cup \tau 6(\mathrm{Z}) \cup \tau 8(\mathrm{Z}) \cup \tau 9(\mathrm{Z})$ $\cup \tau 12(\mathrm{Z}) \cup \tau 13(\mathrm{~T})$

The first results on coding this is a set $\mathrm{X}$ to be an encoding of some of the set can be determined by equations of the form $\mathrm{X}+\mathrm{C}=\mathrm{D}$.

A Set $\mathrm{S} \subseteq \mathrm{Z}$ satisfies the equation:

$$
S+\{0,4,11\}=\bigcup_{i \in\{0,1,3,4,6,7,8,9,10,12,13\}} \tau_{i}(Z) \cup\{11\}
$$

if and only if $\mathrm{S}=\sigma(\mathrm{T})$ for some $\mathrm{T} \subseteq \mathrm{Z}$.

Sketch Of Proof. Suppose that every set $\mathrm{S}$ satisfies the equation. The first track is empty of $\mathrm{S}$ can be determined by using the properties of $S+\{0,4,11\}$ has the vacant track 2, 5, 14 and 15. With the same argument as in lemma $1, \mathrm{~S}$ has empty tracks $\{2,5,14,15\}-\{0,4,11\}=\{1,2,3,4,5,7$, $10,11,14,15\}$. Then the same analysis is applied to track track 11 from $S+\{0,4,11\}$. Each track $\mathrm{t}$ of $\mathrm{S}$ with $\mathrm{t}+\mathrm{tJ}=11$ for some $\mathrm{tJ}$ $\in\{0,4,11\}$ must be empty or the code $\{0\}$ and the latter should be the smallest has at least one $t$. This results in that the track 0 of the $\mathrm{S}$ encoding $\{0\}$.

As a last step, it is seen that $S$ has a track full of. For this purpose, a track full of $\{3,7,9,10\}$ of $S+\{0,4,11\}$ has been investigated. Each track $\mathrm{t}$ is a combination of the track $\mathrm{t}-\{0,4,11\}$ of $\mathrm{S}$. It turns out that in each case two track is already known empty and thus it can be concluded that the track $\{6,8,9,12\}$ of $S$ is full.

To show that $\mathrm{S}=\sigma(\mathrm{T})$ for some $\mathrm{T} \subseteq$ $\mathrm{Z}$ satisfies the equation:

$S+\{0,4,11\}={ }_{i}^{S} \in\{0,1,3,4,6,7,8,9$, $10,12,13\} \tau_{i}(Z) \cup\{11\}$

the set $\sigma(\mathrm{T})$ is represented as a composite of $\sigma(\mathrm{T})=\mathrm{U} 15 \operatorname{ti}(\operatorname{TRACKi}(\sigma(\mathrm{T})))$ and these 
representatives proceed on $\sigma(\mathrm{T})+\{0,4$, 11\} :

$$
\begin{aligned}
& \sigma(T)+\{0,4,11\}=\cup_{i} \tau_{i}\left(T R A C K_{i}(\sigma(T\right. \\
& ))+0) \cup \tau_{i}\left(T R A C K_{i}(\sigma(T))+4\right) \cup \\
& \tau_{i}\left(T R A C K_{i}(\sigma(T))+11\right)
\end{aligned}
$$

As with previous calculations obtained results are given in table 4.1, where the last column of the table contains the results of the calculation of the overall and it can be seen that each copy of $\mathrm{T}$ in a joint replaced by some of the tracks in full.

Now, with the assumption that the system equation with combined and sum have the equation in the form $\mathrm{X}=\mathrm{U}+\mathrm{V}, \mathrm{X}$ $=\mathrm{U} U \mathrm{~V}$ or $\mathrm{X}=$ constant, this equation can

\begin{tabular}{|c|c|c|c|c|c|}
\hline & $\begin{array}{c}\sigma(T) \\
\{0\}\end{array}$ & $\frac{\sigma(T)+\{0\}}{\{0\}}$ & $\frac{\sigma(T)+\{4\}}{7}$ & $\sigma(T)+\{11\}$ & $\sigma(T)+\{0,4,11\}$ \\
\hline $\begin{array}{l}1 \\
2 \\
3 \\
4\end{array}$ & & $\{0\}$ & $\begin{array}{c}\mathrm{N} \\
\mathrm{T}+1 \\
\mathrm{Z}+1 \\
\{0\}\end{array}$ & $\begin{array}{l}\mathrm{N}+1 \\
\mathrm{Z}+1 \\
\mathrm{Z}+1\end{array}$ & $\begin{array}{c}\stackrel{N}{+}+1 \\
Z\end{array}$ \\
\hline 6 & Z & Z & & & $\mathrm{Z}$ \\
\hline 8 & Z & $\mathrm{Z}$ & & $\mathrm{T}+1$ & $\mathrm{Z}$ \\
\hline $\begin{array}{l}10 \\
11\end{array}$ & & & Z & $\{0\}$ & $\begin{array}{c}\bar{Z} \\
\{0\}\end{array}$ \\
\hline $\begin{array}{l}12 \\
13 \\
14 \\
15\end{array}$ & ${ }_{\mathrm{T}}^{\mathrm{Z}}$ & ${ }_{\mathrm{T}}^{\mathrm{Z}}$ & $\begin{array}{l}\mathrm{Z} \\
\mathrm{Z}\end{array}$ & & $\begin{array}{l}\mathrm{Z} \\
\mathrm{Z}\end{array}$ \\
\hline
\end{tabular}
be simulated in the new system as follows:

Table 4.1. Track in $\sigma(\mathrm{T})+\{0,4,11\}$

Table 4.2. Track the number of $\sigma(U)+\sigma(V)$

\begin{tabular}{ccccccc}
\hline & $0:\{0\}$ & $6: \mathrm{N}$ & $8: \mathrm{N}$ & $9: \mathrm{N}$ & $12: \mathrm{N}$ & $13: \mathrm{V}$ \\
\hline $0:\{0\}$ & $0:\{0\}$ & $6: \mathrm{Z}$ & $8: \mathrm{Z}$ & $9: \mathrm{Z}$ & $12: \mathrm{Z}$ & $13: \mathrm{V}$ \\
$6: \mathrm{Z}$ & $6: \mathrm{Z}$ & $12: \mathrm{Z}$ & $14: \mathrm{Z}$ & $15: \mathrm{Z}$ & $2: \mathrm{Z}$ & $3: ?$ \\
$8: \mathrm{Z}$ & $8: \mathrm{Z}$ & $14: \mathrm{Z}$ & $0: \mathrm{Z}$ & $1: \mathrm{Z}$ & $4: \mathrm{Z}$ & $5: ?$ \\
$9: \mathrm{Z}$ & $9: \mathrm{Z}$ & $15: \mathrm{Z}$ & $1: \mathrm{Z}$ & $2: \mathrm{Z}$ & $5: \mathrm{Z}$ & $6: ?$ \\
$12: \mathrm{Z}$ & $12: \mathrm{Z}$ & $2: \mathrm{Z}$ & $4: \mathrm{Z}$ & $5: \mathrm{Z}$ & $8: \mathrm{Z}$ & $9 \vdots ?$ \\
$13: \mathrm{U}$ & $13: \mathrm{U}$ & $3: ?$ & $5: ?$ & $6: ?$ & $9: ?$ & $10:(U+V)+1$ \\
\hline
\end{tabular}

For all of the set $\mathrm{X}, \mathrm{U}, \mathrm{V} \subseteq \mathrm{Z}$, $\sigma(\mathrm{U})+\sigma(\mathrm{V})+\{0,1\}=\sigma(\mathrm{X})+\sigma(\{0\})+$ $\{0,1\}$ if and only if $\mathrm{U}+\mathrm{V}=\mathrm{X} \sigma(\mathrm{U})+\sigma(\mathrm{V}$ )$+\{0,2\}=\sigma X+\sigma(X)+\{0,2\}$ if and only if $\mathrm{U} U \mathrm{~V}=\mathrm{X}$

Sketch of the proof. Seen that for all $\mathrm{U}, \mathrm{V} \subseteq \mathrm{Z}$ the number of $\sigma(\mathrm{U})+\sigma(\mathrm{V})$ encoding the set $U+V+1$ and $U U V$ in some of the tracks. The second encoding is obtained uniquely by adding constants $\{0$, $1\},\{0,2\}$. $\sigma(\mathrm{U})=(\{0\}) \cup \tau 6(\mathrm{Z}) \cup \tau 8(\mathrm{Z}) \cup \tau 9(\mathrm{Z}) \cup$ $\tau 12(\mathrm{Z}) \cup \tau 13(\mathrm{U})$

and

$\sigma(\mathrm{V})=(\{0\}) \cup \tau 6(\mathrm{Z}) \cup \tau 8(\mathrm{Z}) \cup \tau 9(\mathrm{Z}) \cup$ $\tau 12(\mathrm{Z}) \cup \tau 13(\mathrm{~V})$.
This number can be represented as a composite of 36 track is not empty, is given in table 4.2. The question mark indicates the track with a value that is not specified. The value of each track in $\sigma(\mathrm{U})+\sigma(\mathrm{V})$ is given in the column in table 4.3.

Coding in the set of $\sigma(\mathrm{U})+\sigma(\mathrm{V})$ allows to calculate the encoding set on the track of $\sigma(\mathrm{U})+\sigma(\mathrm{V})+\{0,1\}$ which is done in table 4.3. The result is that for all $U$ and $\mathrm{V}$,

$\operatorname{TRACK} 11(\sigma(\mathrm{U})+\sigma(\mathrm{V})+\{0,1\})=\mathrm{U}+\mathrm{V}$ $+1$

$\operatorname{TRACKi}(\sigma(\mathrm{U})+\sigma(\mathrm{V})+\{0,1\})=\mathrm{Z}$ for $\mathrm{i}$ $I=11$ 


\begin{tabular}{cccccc}
\hline & $\sigma(U)$ & $\sigma(V)$ & $\sigma(U)+\sigma(V)$ & $\sigma(U)+\sigma(V)+\{0,1\}$ & $\sigma(U)+\sigma(V)+\{0,2\}$ \\
\hline 0 & $\{0\}$ & $\{0\}$ & $\mathrm{Z}$ & $\mathrm{Z}$ & $\mathrm{Z}$ \\
1 & & & $\mathrm{Z}$ & $\mathrm{Z}$ & $\mathrm{Z}$ \\
2 & & & $?$ & $\mathrm{Z}$ & $\mathrm{Z}$ \\
3 & & & $\mathrm{Z}$ & $\mathrm{Z}$ & $\mathrm{Z}$ \\
4 & & & $\mathrm{Z}$ & $\mathrm{Z}$ & $\mathrm{Z}$ \\
5 & & & $\mathrm{Z}$ & $\mathrm{Z}$ & $\mathrm{Z}$ \\
6 & $\mathrm{Z}$ & $\mathrm{Z}$ & $\mathrm{Z}$ & $\mathrm{Z}$ \\
7 & & & $\mathrm{Z}$ & $\mathrm{Z}$ & $\mathrm{Z}$ \\
8 & $\mathrm{Z}$ & $\mathrm{Z}$ & $\mathrm{Z}$ & $\mathrm{Z}$ & $\mathrm{Z}$ \\
9 & $\mathrm{Z}$ & $\mathrm{Z}$ & $U+V+1$ & $U+V+1$ & $\mathrm{Z}$ \\
10 & & & & $\mathrm{Z}$ & $\mathrm{Z}$ \\
11 & & & $\mathrm{Z}$ & $\mathrm{Z}$ & $\mathrm{Z} V$ \\
12 & $\mathrm{Z}$ & $\mathrm{Z}$ & $U \cup V$ & $\mathrm{Z}$ \\
13 & $\mathrm{U}$ & $\mathrm{V}$ & $U \cup \mathrm{Z}$ & $\mathrm{Z}$ & $\mathrm{Z}$ \\
14 & & & $\mathrm{Z}$ & & \\
15 & & & & &
\end{tabular}

so

$\mathrm{X}+\mathrm{U}=\mathrm{V}$

true if and only if

$\sigma(\mathrm{X})+\sigma(\{0\})+\{0,1\}=\sigma(\mathrm{U})+\sigma(\mathrm{V})+$ $\{0,1\}$

To set $\sigma 13(\mathrm{U})+\sigma(\mathrm{V})+\{0,2\}$, in the same way

TRACK13( $\sigma((\mathrm{U})+\sigma(\mathrm{V})+\{0,1\}))=\mathrm{U} U$ $\mathrm{V}$

$\operatorname{TRACKj}(\sigma((\mathrm{U})+\sigma(\mathrm{V})+\{0,1\}))=\mathrm{Z}$ for $\mathrm{i}$ $I=13$

and therefore for all $\mathrm{X}, \mathrm{U}, \mathrm{V}$,

$\mathrm{X}=\mathrm{U} \cup \mathrm{V}$

if and only if

$\sigma(\mathrm{X})+\sigma(\mathrm{X})+\{0,2\}=\sigma(\mathrm{U})+\sigma(\mathrm{V})+\{0$, $2\}$.

For each set of hyper-aritmatikal $\mathrm{S}$ $\subseteq \mathrm{Z}$, there is a system of equations in the set of integers that uses the operations of addition and constants periodic has a unique solution with $X 1=T$, where $S=\{n \mid 16 n \in T$ \}.

The constants used in this form $\{0$, $1\}, \sigma(\{0\}), \sigma(\{1\}), \sigma(\mathrm{N}), \sigma(-\mathrm{N})$.

Proof. A system of equations with a combination of and summation represents $\mathrm{S}$. This system is a first for all equations of the form $\mathrm{X}=\mathrm{U}+\mathrm{V}, \mathrm{X}=\mathrm{U} \cup \mathrm{V}$ or $\mathrm{X}=\mathrm{C}$. For each variable $X^{J}$ of this system, the new system has a variable XJ. Further, the equation $\mathrm{U}+\mathrm{V}=\mathrm{X}, \mathrm{U} \cup \mathrm{V}=\mathrm{X}$ or $\mathrm{X}=\mathrm{C}$ be transformed into the equation:
$U^{J}+V^{J}+\{0,1\}=X^{J}+\sigma(\{0\}), U^{J}$ $+V^{J}+\{0,2\}=X^{J}+X^{J}+\{0,1\}$ dan $X^{J}=\sigma(C)$, and the resulting system must have a solution that is unique with $X^{J}=\sigma(\mathrm{X})$. With this system, which is formed is a compilation of $\sigma(\mathrm{S})$, and the summation of equations of extra $\mathrm{X} 1=\mathrm{X}+\{-13\}$ produces the repertoire of $T=\sigma(S)-13$.

\section{System with a Constant Limited}

Will now be shown that the use of constants required and the system of equations using only addition and constants unlimited could not determine the set of infinite rather simple, both in terms of the set of natural numbers or in the case of the set of integers.

This is done by showing that every solution of $(\ldots, S, \ldots)$ the system each component of the infinite can be replaced by the empty set.

Given the system of equations the set of natural numbers or the set of integers, in a variable $(\ldots, \mathrm{Xj}, \ldots, \mathrm{Yi}, \ldots)$ using the sum and constant limited, the system has the solution $(\ldots, \mathrm{Fj}, \ldots, \mathrm{Si}, \ldots)$ where each $\mathrm{Fj}$ is limited and each $\mathrm{Si}$ is not limited, then the $(\ldots, \mathrm{Fj}, \ldots, \phi, \ldots)$ is a solution also.

Proof. Suppose that $(\ldots, \mathrm{Fj}, \ldots, \mathrm{Si}, \ldots)$ is a solution and substituted into each equation: $\phi(\ldots, \mathrm{Fj}, \ldots, \mathrm{Si}, \ldots)=\psi$.

If the two sides produces a set is finite, this means that either $\phi$ or 
$\psi$ refers to each variable $\mathrm{Yi}$. In connection with the substitution of $\mathrm{Xj}=\mathrm{Fj}, \mathrm{Yi}=\phi$ produce the same value from both sides.

Finally, the assumption of the substitution produces an infinite set. Because there are no constants of the infinite and all the $\mathrm{Xj}$ has limited value, this means that each party contains multiplevariable $\mathrm{Y}$. Therefore, by substitution: $\mathrm{Xj}=\mathrm{Fj}, \mathrm{Yi}=\varnothing$, both parties refer to $\phi$.

Note that the system of equations a set of numbers in a variable $(\ldots, \mathrm{Xj}, \ldots, \mathrm{Yi}, \ldots)$ using the sum and constant limited. If it has a solution $(\ldots, \mathrm{Fj}, \ldots, \mathrm{Si}, \ldots)$ where each $\mathrm{Fj}$ is limited and each $\mathrm{Si}$ is not limited then $(\ldots, F j, \ldots, S i+N, \ldots)$ is a great solution.

Proof. As in lemma before, suppose that $(\ldots, \mathrm{Xj}, \ldots, \mathrm{Yi}, \ldots)$ be a solution, each substituted into the equation $\phi(\ldots, \mathrm{Fj}, \ldots, \mathrm{Si}, \ldots)=\psi$.

If both parties refers to $\phi$ or on some set of non-empty finite, this case can be solved by assuming that the value of both parties under substitutable $\mathrm{Xj}=\mathrm{Fj}, \mathrm{Yi}=\mathrm{Si}$ is an infinite set $\mathrm{S}$. Then both sides should contain multiple occurrences of the variable Y. Then substitute $\mathrm{Xj}=\mathrm{Fj}, \mathrm{Yi}=\mathrm{Si}+\mathrm{N}$ which produces the $\mathrm{S}+\mathrm{N}$ on both parties. This will complete the proof that the $(\ldots, \mathrm{Fj}, \ldots, \mathrm{Si}+\mathrm{N}, \ldots)$.

If the system of equations with the set of integers is not known $(\ldots, \mathrm{Xj}, \ldots, \mathrm{Yi}, \ldots)$ using the sum and constant limited has a solution $(\ldots, \mathrm{Fj}, \ldots, \mathrm{Si}, \ldots)$ where each $\mathrm{Fj}$ is limited and each $\mathrm{Si}$ is not limited then $(\ldots, \mathrm{Fj}, \ldots, \mathrm{Z}, \ldots)$ is a great solution.

If the system of equations the set of natural numbers using sum and constant limited has the solution $(\ldots, \mathrm{Si}, \ldots$,$) where$ each $\mathrm{Si}$ is restricted numbers. For a system of equations in the set of integers using addition and constants that are limited, if it has at least a solution $(\ldots, \mathrm{Si}, \ldots$,$) where each$ $\mathrm{Si}$ is restricted numbers (numbers limited or $\mathrm{Z})$.

\section{CONCLUSIONS}

General evidence of the equations in this paper using a system of equations with the form $\mathrm{X}=\mathrm{Y} \mathrm{Z}$ and $\mathrm{X}=\mathrm{C}$ with an alphabet $\Sigma=\{$ a $\}$ with a constant periodic $\mathrm{C}$ $\subseteq$ a*. This result in particular, has implications on the latest research on the equation of the language, in which the repeated occurrences can be replaced by other operations. Most of the operations with repeated events have shown the completeness of the calculation of some of the assumptions of the previous equation of the language.

In this paper there are still some shortcomings, namely lack of a simple example of the system of equations in the set of natural numbers with addition and constants periodic representing each association is not periodic. The reason is that the method is given to establish such a system depends on the first form of the system with combined and sum. While for the next one with a simple example that represents the set is not a periodic need hundreds of variables. If the subsequent use of the coding up to 16 tracks, the result will not be intuitive. With a simple example of the system of equations is not periodic is unique, perhaps a solution will lead to a better understanding.

\section{Acknowledgement: None}

\section{Conflict of Interest: None}

\section{Source of Funding: None}

\section{REFERENCES}

1. Calkin. N. J. dan Erdos. P. (1965.) On a class of aperiodic sum-free sets. Math. Proc. Camb.

2. Phil. Soc. Santa Barbara, USA, 2629, 299-345.

3. Erdos. P. (1965). Complexity of solutions of equations over sets of natural numbers. Annual Symposium on Theoretical Aspects of Computer Science (STACS). Bordeaux, France, 2123 February, 373383.

4. Jez. A. dan Okhotin. A. (2008). On the computational completeness of equations over sets of natural numbers. Automata, Languages and Programming ICALP. Reykjav, Iceland, July, part II, LNCS 5126, 63-74. 
5. Jez. A. dan Okhotin. A. (2009). Onenonterminal conjunctive grammars over a unary alpha- bet”. Computer Science in Russia (CSR). Novosibirsk, Russia, LNCS 5675, 191-202.

6. Kunc. M. (2007). What do we know about language equations?, Developments in Language Theory (DLT 2007, Turku, Finland, July 36, 2007), LNCS 4588, 23-27.

7. Leiss. E. L. (1994). Unrestricted complementation in language equations over a one-letter alphabet. Theoretical Computer Science, 132 , 71-93.

8. Luczak. T. (1995). A note on the density of sum-free sets. J. Combin. Theory Ser. A 70, 334-336.

9. Okhotin. A. (2001). Conjunctive grammars. Journal of Automata,
Languages and Combi- natorics. 6:4, 519535.

10. Sandor. C. (2004). On language equations with complementation. Developments in Language Theory, Santa Barbara, USA, June 2629, LNCS 4036, 420432.

11. Szemeredi. E. (1975). Conjunctive grammars can generate non-regular unary languages. International Journal of Foundations of Computer Science, 19:3 , 597-615.

How to cite this article: Tulus Nadapdap, Tulus, Opim Salim. The equation of the set of natural numbers just to sum. International Journal of Research and Review. 2021; 8(5): 379-388. DOI: https://doi.org/10.52403/ijrr.20210547 